\title{
A Study of the Protective Effect of Bushen Huoxue Prescription on Cerebral Microvascular Endothelia Based on Proteomics and Bioinformatics
}

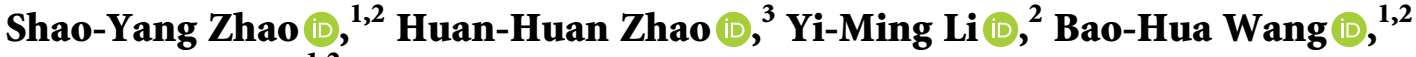 \\ and Sai-Mei Li (DD ${ }^{1,2}$ \\ ${ }^{1}$ Department of Endocrinology, The First Affiliated Hospital of Guangzhou University of Chinese Medicine, Guangzhou 510405, \\ Guangdong, China \\ ${ }^{2}$ The First School of Clinical Medicine, Guangzhou University of Chinese Medicine, Guangzhou 510405, Guangdong, China \\ ${ }^{3}$ Nutrition Department, Linyi People's Hospital, Linyi 276000, Shandong, China
}

Correspondence should be addressed to Bao-Hua Wang; wangbaohua@gzucm.edu.cn and Sai-Mei Li; lsm@gzucm.edu.cn

Received 11 June 2021; Revised 27 November 2021; Accepted 17 December 2021; Published 6 January 2022

Academic Editor: Xue-Rui Wang

Copyright (c) 2022 Shao-Yang Zhao et al. This is an open access article distributed under the Creative Commons Attribution License, which permits unrestricted use, distribution, and reproduction in any medium, provided the original work is properly cited.

\begin{abstract}
Diabetic cognitive dysfunction is a serious complication of type 2 diabetes mellitus (T2DM), which can cause neurological and microvascular damage in the brain. At present, there is no effective treatment for this complication. Bushen Huoxue prescription (BSHX) is a newly formulated compound Chinese medicine containing 7 components. Previous research indicated that BSHX was neuroprotective against advanced glycosylation end product (AGE)-induced PC12 cell insult; however, the effect of BSHX on AGE-induced cerebral microvascular endothelia injury has not been studied. In the current research, we investigated the protective effects of BSHX on AGE-induced injury in bEnd.3 cells. Our findings revealed that BSHX could effectively protect bEnd. 3 cells from apoptosis. Moreover, we analyzed the network regulation effect of BSHX on AGE-induced bEnd. 3 cells injury at the proteomic level. The LC-MS/MS-based shotgun proteomics analysis showed BSHX negatively regulated multiple AGE-elicited proteins. Bioinformatics analysis revealed these differential proteins were involved in multiple processes, such as Foxo signaling pathway. Further molecular biology analysis confirmed that BSHX could downregulate the expression of FoxO1/3 protein and inhibit its nuclear transfer and inhibit the expression of downstream apoptotic protein Bim and the activation of caspase, so as to play a protective role in AGE-induced bEnd.3 injury. Taken together, these findings demonstrated the role of BSHX in the management of diabetic cerebral microangiopathy and provide some insights into the proteomics-guided pharmacological mechanism study of traditional Chinese Medicine.
\end{abstract}

\section{Introduction}

Diabetes mellitus (DM) is a common metabolic disease that can be accompanied by a variety of complications. Diabetic cognitive dysfunction is a major central nervous system complication of DM, mainly manifested as cognitive impairment and neurodegeneration [1-3]. Although the pathogenesis of this complication is still not clear, recent studies have found that overproduction of advanced glycosylation end products (AGEs) is closely related to the occurrence of cognitive dysfunction $[4,5]$. AGEs are toxic substances produced by nonenzymatic glycosylation of proteins and reducing sugars under long-term hyperglycemia. The accumulation of AGEs in the brain can bring about progressive neurostructural changes and functional deficits through different pathways [6-9]. In addition, AGEs can damage cells and even kill them by inducing oxidative stress and reactive oxygen species (ROS) formation $[4,10,11]$. Cerebral microvascular endothelial cells are the basic oxygen supply unit of brains and the blood-brain barrier. In diabetic patients with long-term hyperglycemia, the intense nonenzymatic glycosylation reactions provoke the accumulation 
of AGEs in the vascular endothelium and thus result in vascular endothelial damage and dysfunction [12].

Bushen Huoxue prescription (BSHX) is a newly formulated compound Chinese medicine containing seven components, as listed in Table 1. Previous studies have established that its main components can effectively reduce blood glucose and improve cerebral microcirculatory disturbance and cognitive impairment [13-16]. Icariin (ICA) and Schizandrin $\mathrm{A}$ are the two bioactive ingredients isolated from BSHX. Our previous research results have shown that ICA could specifically bind to the target protein Bax to inhibit its migration to mitochondria, thereby reducing the production of endogenous ROS induced by AGEs and exerting an antiapoptotic effect [17]. In addition, Schizandrin A could alleviate neuron apoptosis and inflammation injury exposed to microglia-conditioned medium. The antiinflammation mechanism of Schizandrin A includes the inhibition of NF- $\kappa \mathrm{B}$ phosphorylation and activation of MAPK pathway. What's more, BSHX has been proven effective clinically in improving diabetic dementia, but it lacks the support of systematic pharmacodynamic evaluation.

Proteomics is a systematic study of the expression, function, and interaction of all proteins in cells. The proteomics technology can reveal the entire protein change spectrum under specific physiological conditions and the effect of drugs on the changes of the whole proteome. Proteomics can be used to analyze the protein differences of diseases before and after drug administration, so as to discover and identify characteristic target proteins. Bioinformatics or systems biology methods can further identify the relevant signal transduction pathways, which can help us explain the pathogenesis of diseases and provide ideas for the diagnosis and treatment of diseases. In this study, the AGEinduced bEnd. 3 cell injury model was used to study the protective effect of BSHX on cerebral microvascular endothelial cells. Combined with proteomics technology, the relevant signal pathways of BSHX inhibiting vascular endothelial injury were analyzed from the overall protein level, so as to reveal the molecular mechanism of BSHX in protecting cerebral microvascular endothelial cells.

\section{Materials and Methods}

2.1. Materials. BSHX granules were purchased from Beijing Kangrentang Pharmaceutical Co. Ltd. (Beijing, China). The medicinal components of BSHX are listed in Table 1. AGEs were purchased from Beijing Biosynthesis Biotechnology Co. Ltd. (Beijing, China) with a purity greater than $95 \%$ by high-performance liquid chromatography (HPLC). Antibodies against cleaved caspase-3, Bim, Histone H3, FoxO1, FoxO3a, $\alpha$-Tubulin, and rabbit IgG were obtained from Cell Signaling Technology (Boston, MA, USA). 3-(4,5-Dimethylthiazol-2-yl)2,5-diphenyltetrazolium bromide (MTT) was bought from Sigma-Aldrich Chemical Co. (Saint Louis, MO, USA). The Dulbecco's modified eagle's medium (DMEM) and fetal bovine serum (FBS) were purchased from PANBiotech GmbH (Aidenbach, Germany). The endothelial cell medium (ECM) was obtained from Beijing M\&C Gene Technology Ltd. (Beijing, China). The lactate dehydrogenase
(LDH) assay kit was purchased from Nanjing Jiancheng Bioengineering Institute (Nanjing, China). Hoechst 33258 was obtained from Beijing Solarbio Science \& Technology Co. Ltd. (Beijing, China). The enhanced BCA protein assay kit was obtained from Beijing TransGen Biotech Co. Ltd. (Beijing, China).

2.2. HPLC Analysis. BSHX extracts were analyzed using a Shimadzu prominence liquid chromatography platform (Kyoto, Japan) equipped with two LC-20AT pumps, CTO20A column oven, DGU-20A5R degasser, SIL-20A autosampler, and SPD20AD detector. Chromatographic separation was conducted on a AichromBond-AQ C18 column $(250 \mathrm{~mm} \times 4.6 \mathrm{~mm}, 5 \mu \mathrm{m}$; Abel Industries Ltd., Canada), protected by a Phenomenex ${ }^{\circledR}$ C18 guard cartridge $(3 \times 4 \mathrm{~mm}, 5 \mu \mathrm{m}$; Torrance, CA, USA). The mobile phase consisted of ACN (A) and $0.1 \%$ aqueous formic acid (B) and was delivered at $1.0 \mathrm{~mL} / \mathrm{min}$ with the following gradient program: $0-35 \mathrm{~min}, \quad 0-3 \% \mathrm{~B} ; \quad 35-100 \mathrm{~min}, \quad 3-22 \% \mathrm{~B}$; 100-115 min, $\quad 22-35 \% \mathrm{~B} ; \quad 115-130 \mathrm{~min}, \quad 35-35 \% \mathrm{~B}$; 130-140 min, $35-100 \% \mathrm{~B} ; 140-145 \mathrm{~min}, 40-100 \% \mathrm{~B}$; and $145-155 \mathrm{~min}, 100-100 \% \mathrm{~B}$. The column was maintained at $40^{\circ} \mathrm{C}$. At the end of each run, the delivery of $100 \%$ A was performed for another $14 \mathrm{~min}$ for system reequilibration. The monitor wavelength was set at $235 \mathrm{~nm}, 254 \mathrm{~nm}$, and $280 \mathrm{~nm}$, respectively.

2.3. Cell Culture. bEnd.3 rat cerebral microvascular endothelial cells were purchased from the Cell Center of the Chinese Academy of Medical Sciences (Beijing, China). Cells were maintained in the endothelial cell medium (ECM) supplemented with $10 \%(\mathrm{v} / \mathrm{v})$ heat-inactivated fetal bovine serum (FBS) and $1 \%(\mathrm{v} / \mathrm{v})$ penicillin-streptomycin (PS) in a humidified $5 \% \mathrm{CO}_{2}$ incubator at $37^{\circ} \mathrm{C}$.

2.4. Sample Treatment. bEnd.3 cells were divided into the control group, model group and BSHX low-dose group, and medium-dose group and high-dose group. First, cells in all groups were cultured to the logarithmic growth phase, then starved with FBS-free ECM for $24 \mathrm{~h}$. Then cells were replaced with the normal ECM containing 10\% FBS for further culture. Afterwards, the cells in the model group were stimulated with $300 \mu \mathrm{g} / \mathrm{mL}$ AGEs for 72 hours; the low-dose group was added with $300 \mu \mathrm{g} / \mathrm{mL}$ AGEs and $20 \mathrm{mg} / \mathrm{L}$ BSHX; the medium-dose group was added with $300 \mu \mathrm{g} / \mathrm{mL}$ AGEs and $50 \mathrm{mg} / \mathrm{L} \mathrm{BSHX}$; and the high-dose group was treated with $300 \mu \mathrm{g} / \mathrm{mL}$ AGEs and $50 \mathrm{mg} / \mathrm{L}$ BSHX for $72 \mathrm{~h}$. The control group was not treated with dosing.

2.5. Cell Viability Assay. bEnd.3 cells were seeded into a 96well plate (3500/well) for $24 \mathrm{~h}$, followed by AGEs $(300 \mu \mathrm{g} / \mathrm{mL})$ and $\operatorname{BSHX}(20,50$, and $100 \mathrm{mg} / \mathrm{L}$, respectively) treatment as mentioned previously. Cell viability was determined using MTT. In brief, add $200 \mu \mathrm{L}$ MTT solution $(0.5 \mathrm{mg} / \mathrm{mL})$ in each well for $4 \mathrm{~h}$ at $37^{\circ} \mathrm{C}$. Then replace the original supernatant with $200 \mu \mathrm{L}$ DMSO and continue to incubate for another $2 \mathrm{~h}$. The optical density (OD) values were measured at $570 \mathrm{~nm}$ using a 
TABle 1: Composition of BSHX.

\begin{tabular}{lccc}
\hline Herbal composition & Chinese name & Part used & Percentage of total weight \\
\hline Cuscuta chinensis Lam. & Tu Si Zi & Fruit & 25 \\
Lycium barbarum L & Gou Qi Zi & Fruit & 25 \\
Rubus chingii Hu. & Fu Pen Zi & Fruit & 12 \\
Schizandra chinensis (Turcz.) Baill. & Wu Wei Zi & Fruit & 3 \\
Plantago asiatica L. & Che Qian Zi & Seed & 6 \\
Epimedium brevicornu Maxim. & Yin Yang Huo & Stem Leaf & 25 \\
Hirudo nipponica Whitman & Shui Zhi & The whole body & 4 \\
\hline
\end{tabular}

microplate reader (Bio-Rad Laboratories Inc., Hercules, CA, USA). Cell viability $(\%)=[\mathrm{OD}$ (treatment) $-\mathrm{OD}$ (blank)]/[OD (control) - OD (blank)] $\times 100 \%$.

2.6. Lactate Dehydrogenase (LDH) Assay. bEnd.3 cells were exposed to AGEs $(300 \mu \mathrm{g} / \mathrm{mL})$ with or without BSHX (20, 50, and $100 \mathrm{mg} / \mathrm{L}$, respectively) as above. Then, use a commercial kit for the detection of LDH release from the cells, according to the manufacturer's instructions. Absorbance was measured at $450 \mathrm{~nm}$. LDH $(\mathrm{U} / \mathrm{L})=[\mathrm{OD}$ (treatment) OD (blank)] $/[\mathrm{OD}$ (standard) - OD (blank) $\times 0.2 \quad(\mathrm{mmol} /$ L) $\times 1000$.

2.7. Hoechst 33258 Staining and Annexin V/PI Staining. Cells were grown on cover glasses (NEST, Wuxi, Jiangsu Province, China) placed at the bottom of a 24 -well plate $(20,000$ cells/well $)$ and subjected to AGEs with or without BSHX as described. After 72 hours, the cell culture medium was removed, and $4 \%$ paraformaldehyde $(500 \mu \mathrm{L} /$ well $)$ was added to each well for $20 \mathrm{~min}$, followed by Hoechst 33258 solution $(1 \mu \mathrm{g} / \mathrm{ml}$ in PBS) staining in the dark for $30 \mathrm{~min}$ at room temperature. Images were captured using a fluorescence microscope (IX73, Olympus, Japan) under an excitation wavelength of $352 \mathrm{~nm}$ and an emission wavelength of $461 \mathrm{~nm}[17,18]$.

For Annexin V/PI staining, bEnd. 3 cells were treated as showed previously incubation in 6-well plates with an initial density of $2.0 \times 10^{5}$ cells per well. All the operations simply followed the instructions. First, harvested cells with the corresponding supernatants. Then, washed them two times with cold Phosphate Buffered Saline (PBS, Gibco) after centrifugation $(500 \times \mathrm{g}, 5 \mathrm{~min})$. Next, cells were suspended in binding buffer and stained with Annexin V-FITC $(5 \mu \mathrm{L}) / \mathrm{PI}$ $(5 \mu \mathrm{L})$ mixture for $15 \mathrm{~min}$ at room temperature. Detected within an hour with BD FACSCelesta (BD Biosciences, NJ, USA), and data were analyzed using FlowJo software (BD, Version: 10.7.2).

2.8. Protein Identification by Nano LC-MS/MS. bEnd. 3 were divided into the control group, model group, and BSHX high-dose $(100 \mathrm{mg} / \mathrm{L})$ group. First, the cells were cultured in ECM containing AGEs $(300 \mu \mathrm{g} / \mathrm{mL})$ with or without BSHX-contained $(100 \mathrm{mg} / \mathrm{L})$ AGEs for $72 \mathrm{~h}$. Then cells were scraped with a spatula and washed by PBS. The cell pellet was extracted with NP40 lysate to obtain total proteins and the protein concentration was determined by the BCA method. After the protein concentration was quantified, SDS-PAGE gel electrophoresis and silver staining were performed. Whole protein bands on SDS-PAGE were excised and digested with trypsin. Afterwards, the obtained peptide mixture was filtered through a $0.22 \mu \mathrm{m}$ micropore membrane to afford mass spectrometry analysis samples. Extracted peptide samples were analyzed by the nano-liquid chromatography coupled with hybrid linear ion trap-Orbitrap mass spectrometer (nanoLC-LTQ-Orbitrap MS/MS) method. The specific operation steps can refer to the previous research of our research group [19].

2.9. Bioinformatics Analysis. Proteins were quantified according to the sum of the signal intensity (the peak area of the precursor) of the first order spectrum of each peptide detected. The proteins with expression difference $>5$ in the control group and model group, as well as the model group and BSHX group, were selected as differential proteins.

The identified differential proteins were uploaded to the Database for Annotation, Visualization, and Integrated Discovery $\left(\right.$ DAVID $\left.^{1}\right)$ and Protein Analysis Through Evolutionary Relationships database $\left(\right.$ PANTHER $^{2}$ ) databases systems, and the gene ontology $(\mathrm{GO})$ protein classification analysis including the cellular component (CC), the molecular function (MF), and the biological process (BP) was performed.

In addition, for signal pathway enrichment analysis, we introduced the identified differential proteins into Cytoscape software (v.3.5.1) and used the attached ClueGO plug-in to analyze the Kyoto Encyclopedia of Genes and Genomes (KEGG), REACTOME pathway, and Wiki pathway. The species was set as mice. $P<0.05$ was set as the significance threshold, and the default parameters were used for the rest.

2.10. Western Blot Analysis. Cells were vortexed in cold RIPA buffer with protease and phosphatase inhibitor (1x) for $30 \mathrm{~min}$. Nuclear and cytoplasmic proteins were prepared using the Nuclear and Cytoplasmic Protein Extraction Kit (Beyotime) as per the manufacturer's protocol. Total proteins were extracted after centrifugation $(12,000 \mathrm{rpm}$, $10 \mathrm{~min}, 4^{\circ} \mathrm{C}$ ), and protein concentrations were determined using a BCA Assay Kit (TransGen Biotech). Protein samples from each group were subjected to $10 \%-15 \%$ SDS-PAGE gels system and subsequently transferred to polyvinylidene fluoride (PVDF) membranes (Millipore, Billerica, MA, USA). The membranes were blocked by $5 \%(\mathrm{w} / \mathrm{v})$ skim milk at room temperature for $60 \mathrm{~min}$, then washed 3 times with 


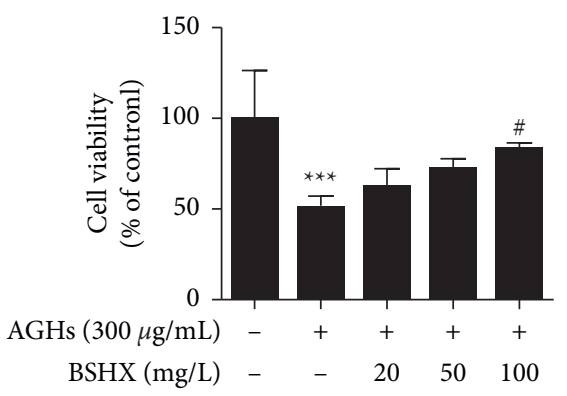

(a)
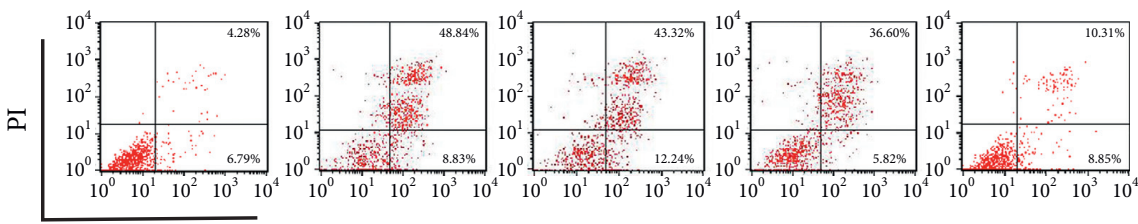

Annexin-V FITC

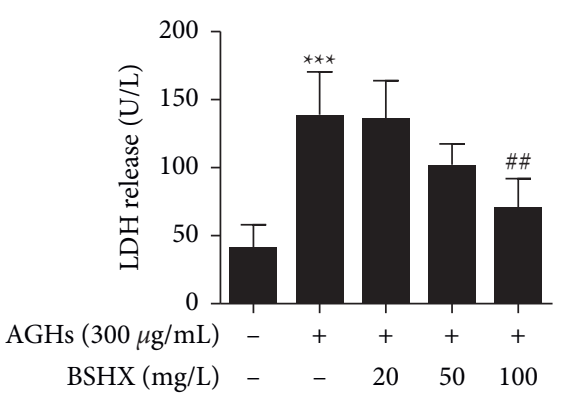

(b)

(c)

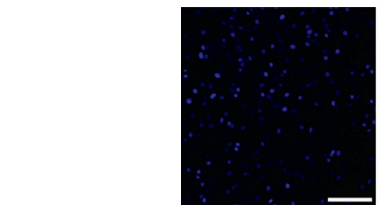

AGEs $(300 \mu \mathrm{g} / \mathrm{mL})$ BSHX (mg/L)

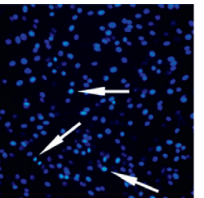

$+$

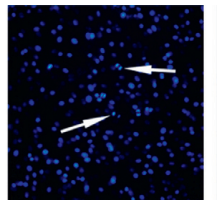

20

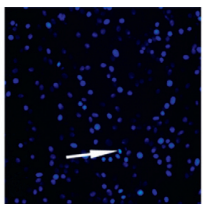

$+$

50

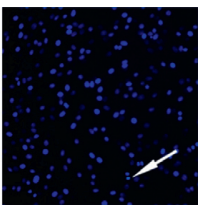

+
100

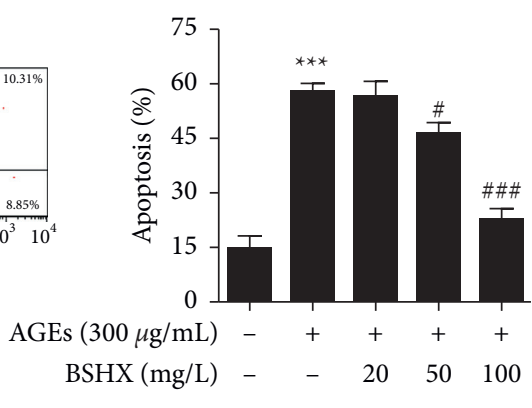
. 
with the control group, AGEs $(300 \mu \mathrm{g} / \mathrm{mL})$ led to a significant cell injury (cell viability decreased to $56.55 \pm 9.7 \%$, $P<0.001)$, while BSHX treatment $(20,50$, and $100 \mathrm{mg} / \mathrm{L})$ restored the cell viability in a dose-dependent manner. Furthermore, we detected the LDH, which released from injured cells, to assess the cell toxicity after drug exposure (Figure 1(b)). We found that LDH released in the supernatant of cells from the model group dramatically swelled to $142.02 \pm 13.77 \mathrm{U} / \mathrm{L}(P<0.001$ vs. Control group $)$ and BSHX $(100 \mathrm{mg} / \mathrm{L})$ significantly shrunk the release and remedied the cytotoxity $(74.83 \pm 9.28 \mathrm{U} / \mathrm{L}, P<0.01$ vs. model group). Briefly, our results indicates that BSHX has an excellent protective effect on damaged bEnd. 3 cells induced by AGEs.

To further verify the effect of BSHX on cell apoptosis, we stained the cells with Annexin V/PI. Translocation of phosphatidylserine occurs in early apoptotic cells whose cytomembranes are stained by Annexin V only. Incomplete cytomembrane of late apoptotic cells results in nuclei staining by PI. Cells stained by both Annexin V/PI are considered necrotic or fragmented. Dot plots (Figure 1(c)) showed percentage of apoptotic cells (early and late apoptotic cells) in each group-It was $11.07 \%$ in the control group, $57.67 \%$ in model group, $55.54 \%$ in the low dose group (n.s.), $42.42 \%$ in medium dose group $(P<0.05$ vs. model group) and $19.16 \%$ in high dose group $(P<0.001$ vs. model group), respectively.

Moreover, to observe apoptosis directly, cells were subjected to Hoechst 33258 staining which can penetrate the cell membrane and release strong blue fluorescence after embedding in the double stranded DNA of apoptotic cells. The results (Figure 1(d)) revealed that AGEs treatment dramatically promoted chromosome condensation $(P<0.001$ vs. Control group), which was a crucial indication of cell apoptosis. BSHX of a concentration of $20-100 \mathrm{mg} / \mathrm{L}$ effectively attenuated that change $(P<0.05$ or $P<0.001$ vs. model group). All of these results suggested that AGEs significantly induced bEnd. 3 cell apoptosis and BSHX could protect bEnd. 3 cells from AGE insults.

\subsection{Effects of BSHX on Proteomic Changes Induced by AGEs in} bEnd. 3 Cells. A quantitative proteomics analysis was carried out using the Nano-LC-MS/MS system to dissect the effect of BSHX on the proteome profile. According to the protein identification results of the bEnd. 3 cell lysates based on the Thermo Proteome Discoverer database, there were a total of 314 differentially expressed proteins between the model group and the control group, and 217 proteins were differentially expressed in the BSHX groups and the model group. The relationship of identified proteins in the control, model, and BSHX groups is shown in the Venn diagram (Figure 2(a)).

Comparing the expression levels of the whole proteins in different groups, the differentially expressed protein candidates were acquired. In this study, proteins with a more than fivefold increase or decrease were filtrated. Finally, 314 differentially expressed proteins were identified. Specifically, 223 proteins were upregulated and 91 were downregulated upon AGEs treatment. These 314 differentially expressed proteins were further analyzed between the model and the BSHX groups. Results showed that the expression levels of 107 (47.9\%) upregulated proteins and 19 (20.9\%) downregulated proteins in the model group were markedly reversed in response to BSHX treatment.

In addition, to explore the impact of BSHX on proteomic changes, the proteins with specific response to BSHX treatment were annotated and categorized according to gene ontology (GO) protein classification analysis (including CC, MF, and BP). As shown in Figure 2(b), these proteins were mainly distributed in organelles or organelle parts (124 downregulated proteins) and the membrane system (77 downregulated proteins). Functionally, they participated in activities as diverse as the binding activity (74 downregulated proteins), the catalytic activity ( 35 downregulated proteins), and the transcription regulation activity (8 downregulated proteins) (Figure 2(c)). Moreover, these proteins were involved in complicated BPs including cellular processes (73 downregulated proteins), biological regulation (61 downregulated proteins), metabolic processes (45 downregulated proteins), and so on. (Figure 2(d)) Taken together, the above-mentioned results indicated that BSHX notably downregulated the expression of AGE-irritated proteins and effectively blocked the related multiple BPs.

\subsection{Bioinformatics Analysis for the BSHX-Regulated Signaling} Networks. To visualize BSHX-regulated pharmacological networks, the 107 proteins downregulated by BSHX were examined for enrichment in KEGG pathway, REACTOME pathway, and Wiki pathway database from ClueGO plug-in. The pathway networks depicting GO terms with a value of $P<0.05$ were mapped in Figure 3(a) and listed in Figure 3(b). Results showed that the FoxO pathway, cell cycle pathway, Hippo pathway, apoptosis, and AGE-RAGE signaling pathway in diabetic complications and neurotrophin signaling pathway were the most enriched pathways. To confirm this, GO Biological Process enrichment analysis related to the above-mentioned functionally annotated proteins was utilized. Result showed that multiple biological processes were significantly enriched, including apoptotic process, positive regulation of Foxo signaling, developmental process, metabolic process, response to stimulus, regulation of immune system process, and cell activation involved in immune response (Figure 4(c)). All these biological processes are related to apoptosis. Together, these findings suggested that BSHX could effectively extinguish multiple AGE-activated pathways and BPs and exerted antiapoptotic activity.

3.5. BSHX Suppressed FoxO1/3 Pathway in AGE-Induced bEnd.3 Cells. FoxOs transcription factors have been revealed to be closely associated with the occurrence and development of diabetes and its complications [20, 21]. In Figure 4(a), the expression of FoxO1 and FoxO3 proteins was significantly enhanced by AGEs stimulation $(P<0.01$ or $P<0.001$ ), while BSHX markedly restrained the overexpression of these two proteins in a concentrationdependent manner $(P<0.01)$ (Figure $4(\mathrm{a}))$. To further 

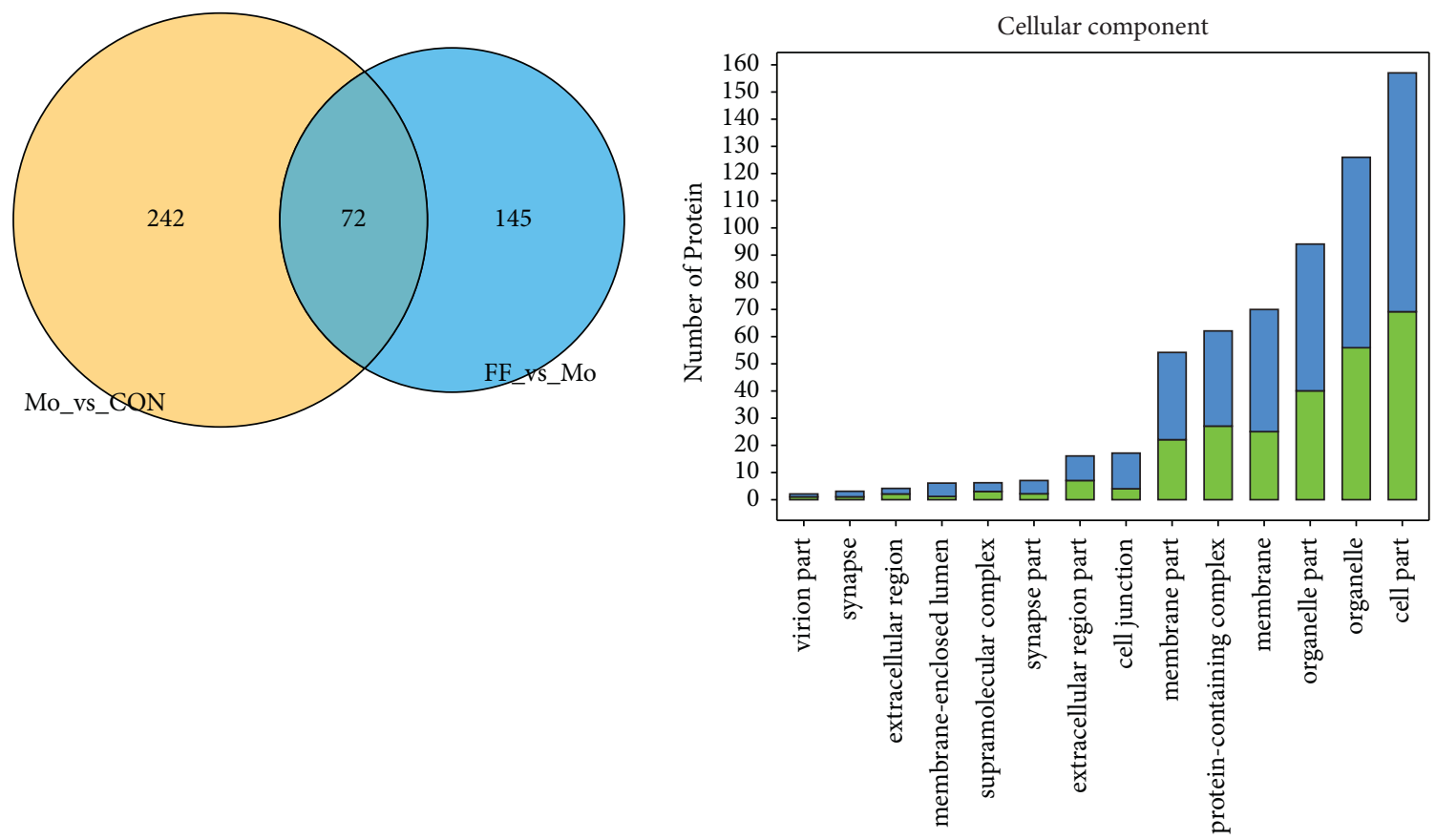

up_down

$\square$ up_regulated

$\square$ down_regulated

(a)
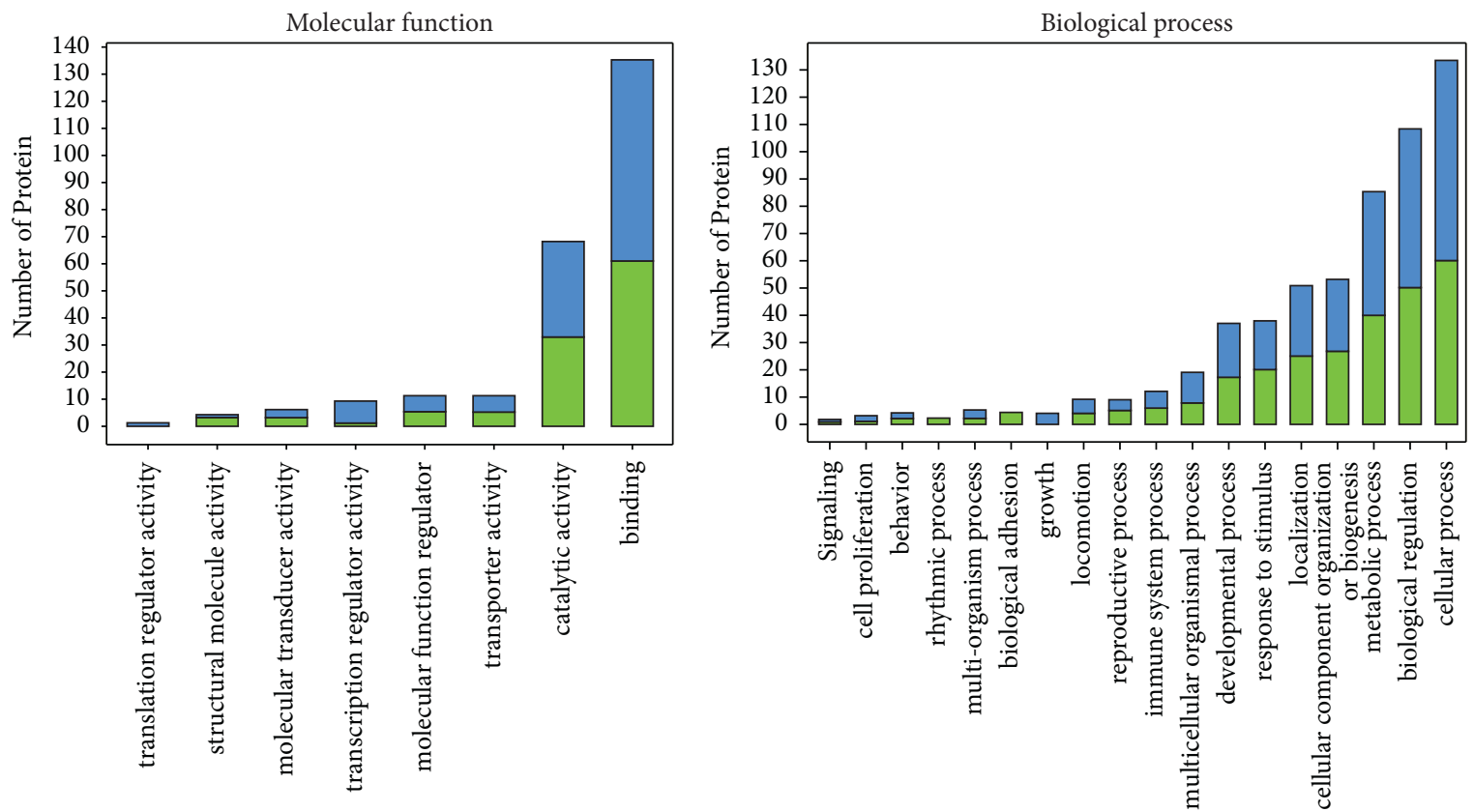

up_down
$\square$ up_regulated
$\square$ down_regulated

up_down

$\square$ up_regulated

down_regulated

(c)

(d)

FIgURE 2: Proteomic analysis results for BSHX-altered proteins. (a) Venn diagram showing numerical distribution of proteins identified in different bEnd. 3 cell lysates by the nanoLC-LTQ-Orbitrap MS/MS approach. Cells were treated with vehicle (control group: Con), $300 \mu \mathrm{g} /$ $\mathrm{mL}$ of AGEs (model group: Mo), and AGEs with $100 \mathrm{mg} / \mathrm{L}$ of BSHX (high-concentration BSHX treatment group: FF) for $72 \mathrm{~h}$ respectively. (b-d) Proteins significantly upregulated and downregulated by BSHX were classified according to the cellular component (b), molecular function (c), and biological process (d). 


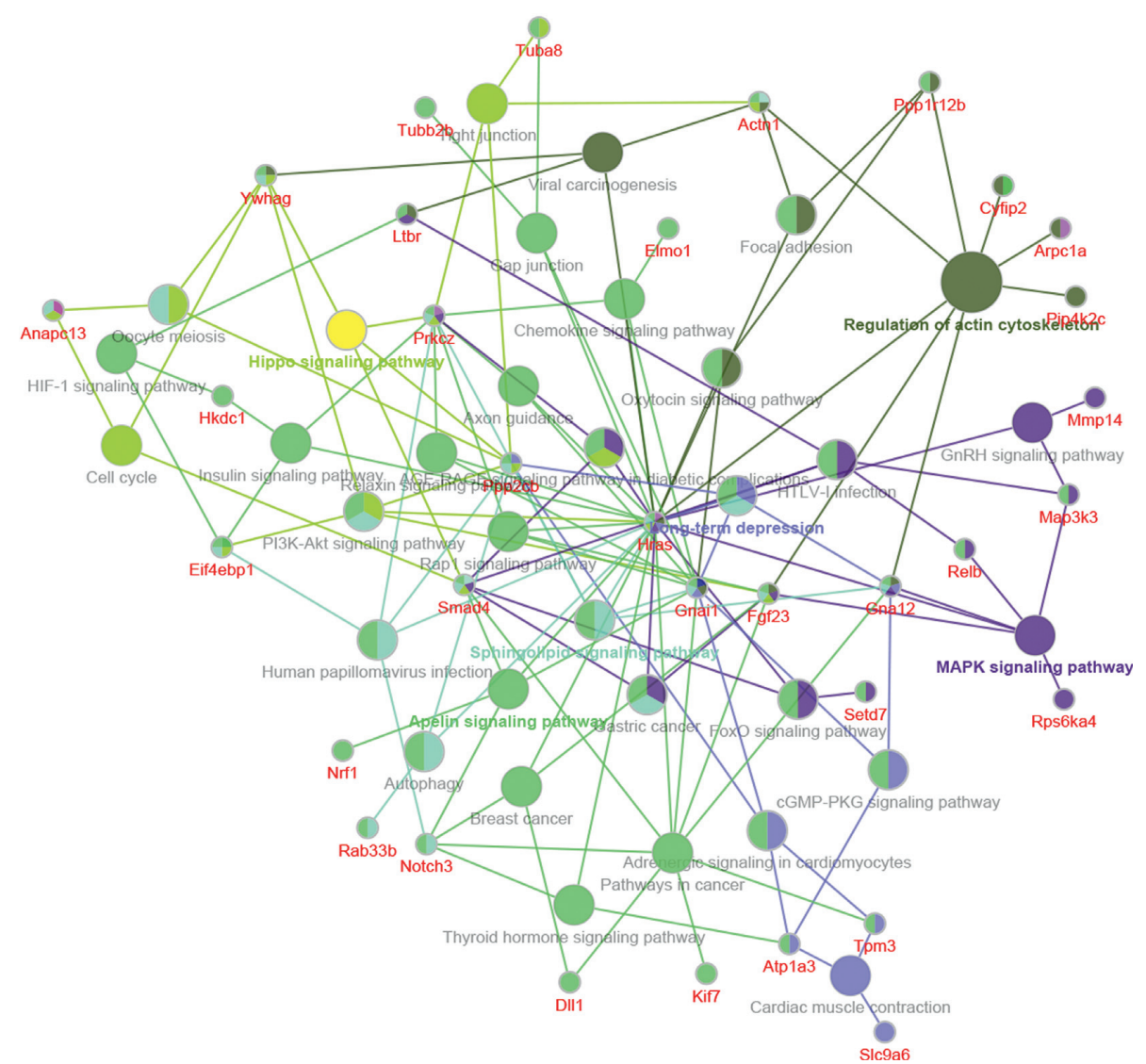

(a)

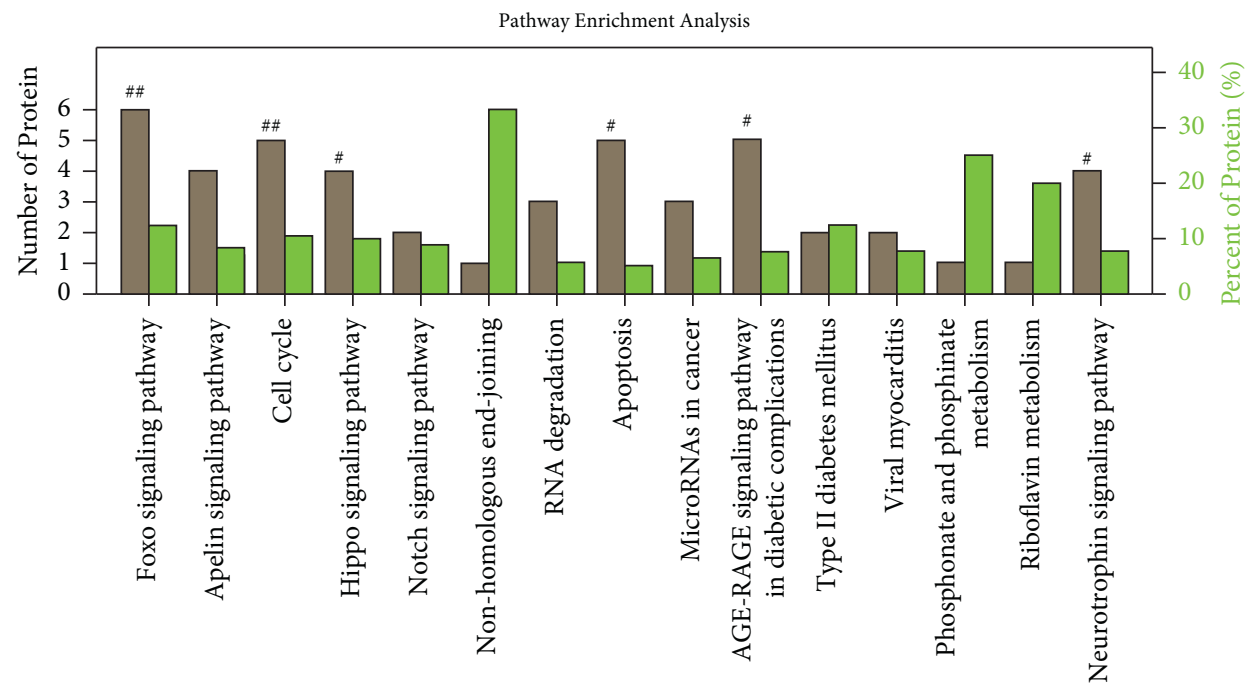

(b)

Figure 3: Continued. 


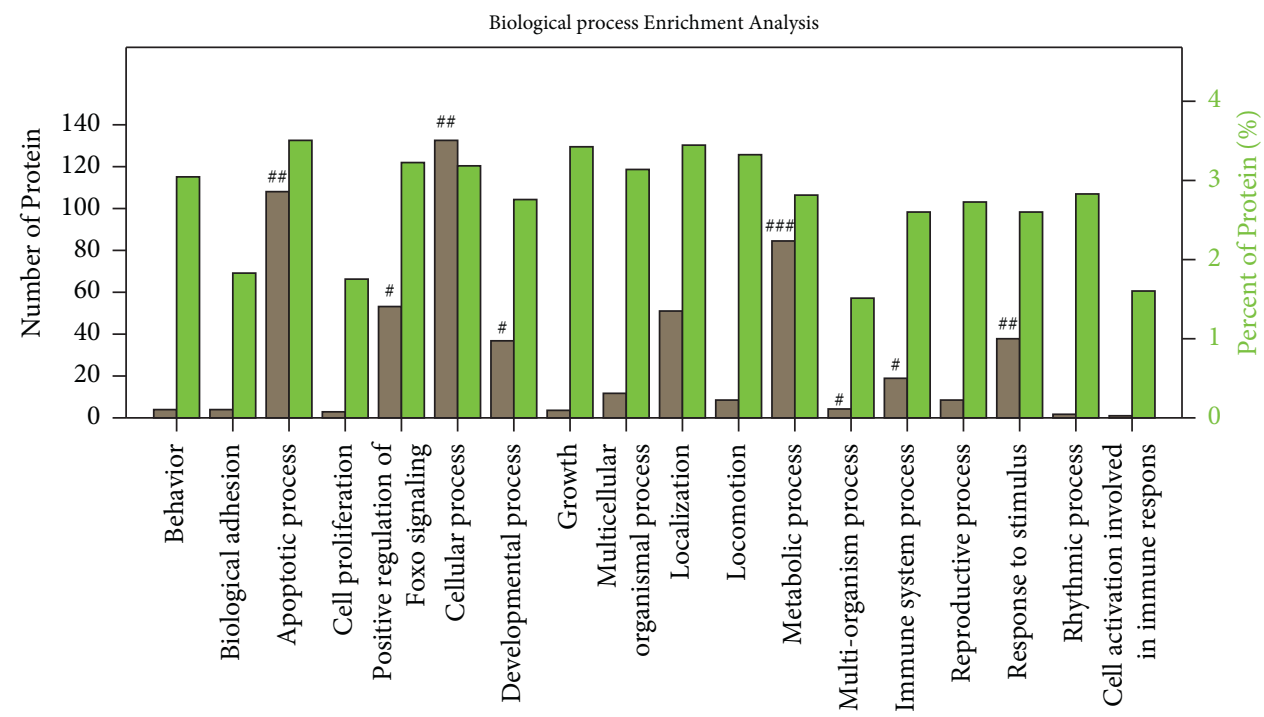

(c)

FIGURE 3: Bioinformatics analysis of the signaling networks negatively regulated by BSHX. (a) Interaction network of protein groups downregulated by BSHX. (b) Pathway enrichment analysis of the differentially expressed proteins predicted the significantly canonical pathways targeted by BSHX in AGE-stimulated bEnd. 3 cells. The gray columns on the left $Y$-axis represent the number of identified proteins in each pathway. The green columns on the right $Y$-axis depict the percentage of identified proteins over the total proteins in that pathway. (c) BP enrichment analysis for differentially expressed proteins in BSHX-treated bEnd. 3 cells. ${ }^{\#} P<0.05,{ }^{\# \#} P<0.01$, ${ }^{\# \# \#} P<0.001$ relative to the model group.
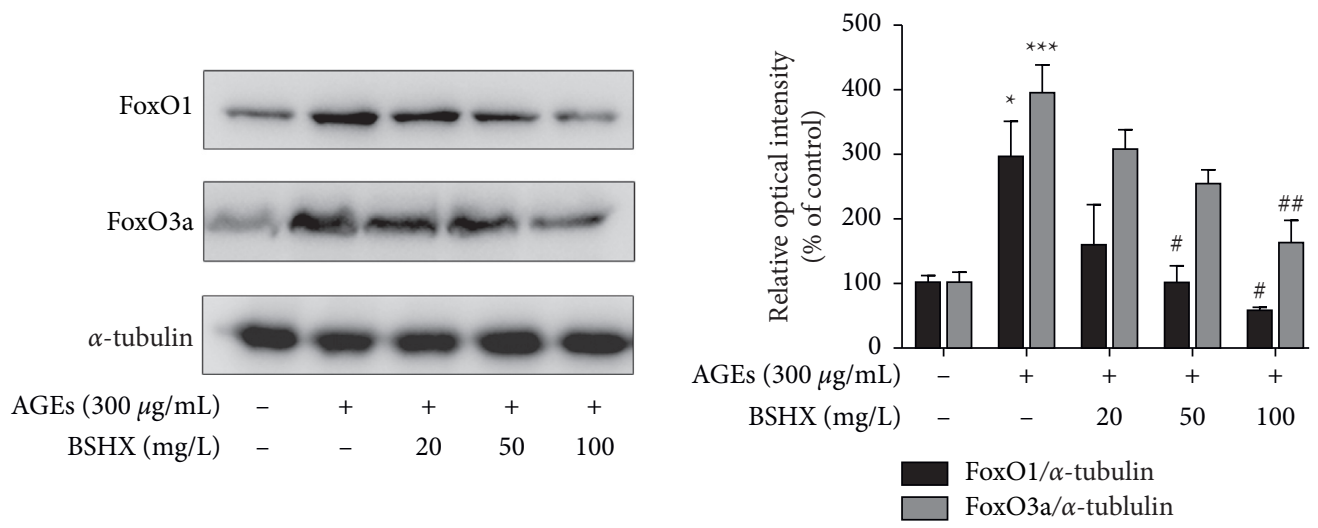

(a)

Figure 4: Continued. 

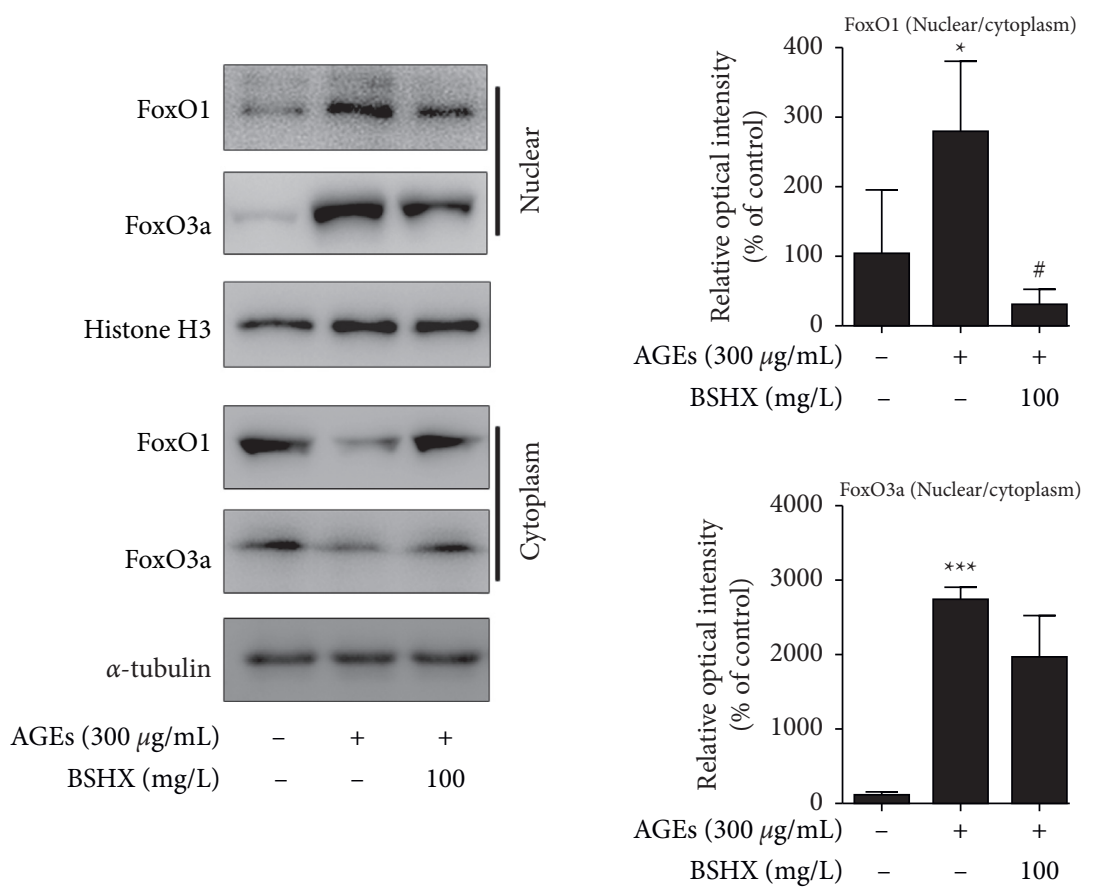

(b)
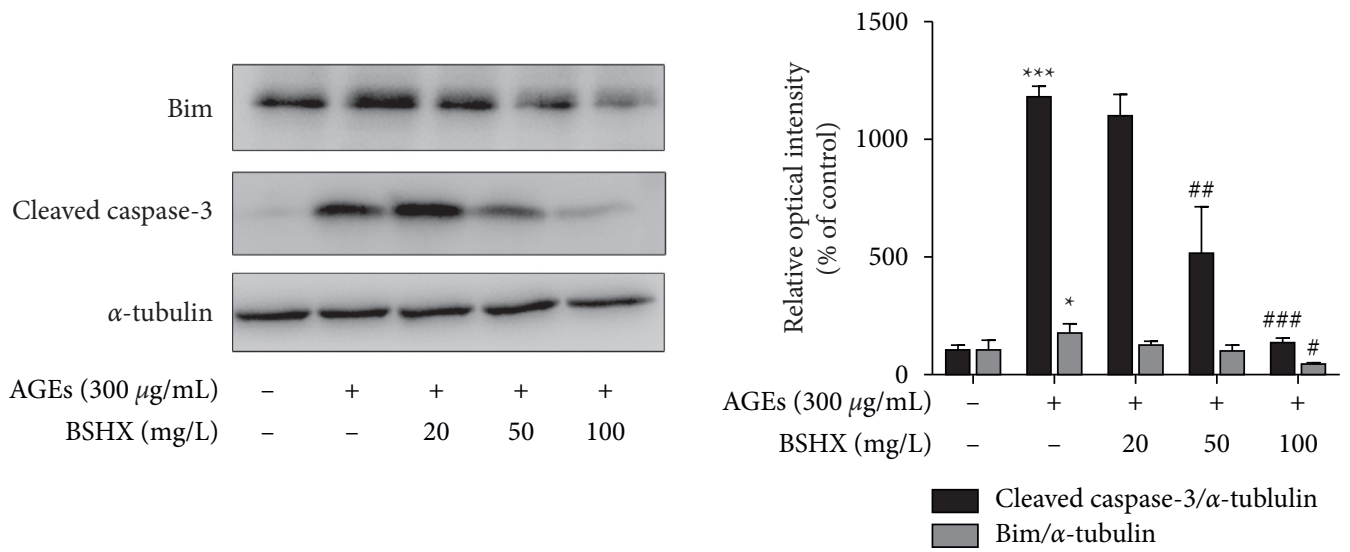

(c)

Figure 4: BSHX prevented FoxO1 and FoxO3 proteins from translocating into the nucleus and promoted their degradation under AGEinduced stimulation. (a) bEnd. 3 cells were treated with AGEs $(300 \mu \mathrm{g} / \mathrm{mL})$ with or without BSHX (20, 50 , and $100 \mathrm{mg} / \mathrm{mL}$, respectively) for $72 \mathrm{~h}$. The total expression of FoxO1 and FoxO3 was determined by Western blot assay. (b) The nuclear and cytoplasmic expression of FoxO1 and FoxO3 in bEnd. 3 cells treated with AGEs $(300 \mu \mathrm{g} / \mathrm{mL})$ with or without BSHX $(100 \mathrm{mg} / \mathrm{mL})$ for $1 \mathrm{~h}$ was determined by Western blot assay. Histone $\mathrm{H} 3$ and $\alpha$-tubulin were utilized as internal controls for nuclear and cytoplasmic proteins, respectively. (c) bEnd. 3 cells were treated as in (a) for $72 \mathrm{~h}$ and then the expression of Bim and cleaved caspase- 3 was detected by Western blot assay. All data were acquired by at least three independent experiments and presented as mean \pm S.D. ${ }^{* *} P<0.01,{ }^{* * *} P<0.001$ relative to the control group; ${ }^{\# \#} P<0.01$, \#\#\# $P<0.001$ relative to the model group.

explore the effect of BSHX on the nuclear translocation of FoxO1/3 that is critical to FoxO1/3-dependent biological processes, the expression of FoxO1 and FoxO3a in the nucleus and cytoplasm of bEnd. 3 cells treated with AGEs and a high dose of BSHX $(100 \mathrm{mg} / \mathrm{L})$ for $1 \mathrm{~h}$ were detected. Results suggested that the expression of FoxO1 and FoxO3a in the nucleus of AGE-challenged bEnd.3 cells was sharply increased $(P<0.001)$, while their expression in the cytoplasm of such cells was decreased (Figure 4(b)). However, $100 \mathrm{mg} / \mathrm{L}$ BSHX markedly blocked the nuclear translocation of FoxO1 and FoxO3a $(P<0.001)$ as demonstrated by the reduced FoxO1/FoxO3a levels in the nucleus and the elevated FoxO1/FoxO3a levels in the cytoplasm (Figure 4(b)). These findings implied that BSHX could inhibit the AGE-induced nuclear transfer of FoxO1/3.

In addition, to further understand the activation level of the FoxO signaling pathway, we also detected the protein expression levels of Bim and caspase-3 as well as the downstream target gene of FoxO transcription factors. It can be seen from 
Figure 4(c) that after stimulating the cells with AGEs for $72 \mathrm{~h}$, the protein levels of Bim and activated caspase- 3 were increased significantly $(P<0.001)$, while BSHX reduced their expression in a dose-dependent manner $(P<0.001)$. These findings indicated that BSHX inhibited the expression and AGE-induced nuclear transfer of FoxO1/3 and downregulated the expression of apoptosis-related proteins Bim and caspase-3, thus arresting the activation of the FoxO pathway and protecting cells from apoptosis.

\section{Discussion}

Cognitive dysfunction is increasingly recognized as one of the most severe complications of diabetes mellitus, which can be manifested as short-term memory and executive function impairment. It has also been acknowledged that diabetes is one of the independent risk factors for cognitive dysfunction. As established by pathological studies, similar to patients with Alzheimer's disease (AD), the brains of diabetes-associated cognitive dysfunction patients are also featured by amyloid deposition and abnormal phosphorylation of tau proteins [22]. What's more, this complication and $\mathrm{AD}$ share many common mechanisms of pathogenesis, such as insulin resistance, cytotoxicity of AGEs, oxidative stress, and inflammation. Therefore, scholars have recently presented a hypothesis that $\mathrm{AD}$ is a kind of type 3 diabetes [23-25].

In our experiments, we studied AGEs, a common pathogenic factor of diabetic cognitive dysfunction and AD. AGEs have cytotoxicity and are a major product of nonenzymatic catalysis reactions of the aldehyde group of glucose and the amino group of proteins. During the normal aging process, AGEs may exist in different cells, but the AGEs levels in patients with diabetes and $\mathrm{AD}$ are significantly increased. In patients with T2DM, hyperglycemia accelerates the accumulation of AGEs in kidneys, retinal vessels, and the central nervous system [26-28]. The combining of AGEs with their receptor RAGE can activate a variety of proinflammatory cytokines and produce a large number of reactive oxygen species (ROS), resulting in mitochondrial dysfunction and cell apoptosis. Recent studies found that the increase of AGEs could cause the hyperphosphorylation of tau proteins and the accumulation of $\mathrm{A} \beta$, thereby accelerating the progress of cognitive impairment and neurodegeneration [29].

BSHX prescription is to add leeches on the basis of the Wuzi Yanzong prescription (Icariside, Cuscuta chinensis, Chinese wolfberry, Rubus idaeus, Schisandra chinensis, plantaginis semen). Leeches have blood stasis removing and menstrual flow restoring effects. BSHX can activate blood circulation to dredge collaterals and tonify the kidney. The clinical application has validated its effectiveness in alleviating memory decline and reducing blood sugars. Apoptosis is a process of programmed cell death, which plays a crucial role in cell death and has been widely studied for neuroprotective agent development [30]. In this study, the neuroprotective effect of BSHX on AGE-induced cytotoxicity and the related potential mechanism were analyzed. The results showed that AGEs insults remarkably reduced the viability of bEnd.3 cells, while BSHX tremendously improved the cell survival rate and inhibited $\mathrm{LDH}$ release in a concentration-dependent manner, confirming the neuroprotective effect of BSHX. In addition, Hoechst 33258 and Annexin V/PI staining results demonstrated that AGEs stimulation induced the fragmented or shrunken nuclei, which, however, were effectively inhibited by BSHX. All in all, BSHX could inhibit bEnd.3 cell apoptosis.

Proteomics is a discipline that studies the qualitative, quantitative, and modified status of all proteins in complete cells, tissues, body fluids, and other samples based on mass spectrometry technology [31]. The research ideas of proteomics are very similar to the holistic and multitarget views of traditional Chinese medicine (TCM). In the study of pharmacology of TCM, proteomics technology can be used to explore the change of protein level of TCM itself. At the same time, we can also perform high-throughput detection of protein expression levels before and after medication, compare the differences in protein expression of cell or tissue samples under different physiological or pathological conditions, and identify and quantify the related proteins, so as to analyze the interactions between proteins and biological functions at a global level. At present, proteomics has successfully revealed the targets and pharmacological mechanisms of many active molecules of traditional Chinese medicine, such as arsenictrioxide, ganoderic acid, and gambogic acid [32-34]. In this study, we applied the integrated proteomics and bioinformatics to identify proteins regulated by BSHX and their biological functions. The LCMS/MS-based shotgun proteomics analysis showed that BSHX negatively regulated multiple AGE-elicited proteins. Bioinformatics analysis revealed that these differential proteins were involved in multiple processes including FoxO pathway, apoptosis, AGE-RAGE signaling pathway in diabetic complications and so on. In addition, multiple Biological Processes, such as apoptotic process, positive regulation of Foxo signaling, metabolic process, regulation of immune system process were significantly enriched. All these findings suggested that BSHX could effectively extinguish multiple AGE-activated pathways and BPs, and exerted antiapoptotic activity.

The FoxO signaling pathway is closely related to the occurrence and development of diabetes and its complications $[35,36]$, and involved in glucose metabolism, apoptosis, inflammation and other biological processes. Exposed to oxidative stress and other harmful stimuli, FoxO proteins in the cytoplasm are dephosphorylated and transferred to the nucleus, where they bind to DNA and promote the expression of downstream apoptotic genes, such as Bim and Fas ligands, consequently initiating the apoptosis [37-39]. Based on this standpoint, the FoxO pathway was studied in this paper, and two most well-understood FoxO proteins, FoxO1 and FoxO3, were further examined to ascertain the detailed mechanism. In line with the proteomic analysis results, Western blot experiments corroborated the inhibitory effects of BSHX on the AGEactivated FoxO pathway. BSHX was shown to regulate the subcellular localization of FoxO1 and FoxO3 proteins, downregulate their protein levels, inhibit their nuclear 
transfer, curb the expression of downstream apoptotic protein Bim, and prevent the activation of caspase. In this regard, our work demonstrated the beneficial role of BSHX in the regulation of the FoxO pathway, and the effectiveness of direct pharmacological inactivation of FoxO1/3 in treating brain microvascular damage in T2DM patients.

In addition, it is noteworthy that there might be some differences between the bEnd. 3 cell line and the primary cells. Although we have identified the BSHX-regulated anticerebral microvascular endothelial injury signaling networks in bEnd.3 cells, a further validation for these above-mentioned crossed signaling pathways in primary cells is warranted and the direct molecular target of BSHX requires identification in future studies.

\section{Conclusions}

In summary, the current study demonstrated the neurovascular protective effect of BSHX on a classical AGEstimulated bEnd.3 cell model and highlighted the related mechanism by an integrated approach of label-free quantitative proteomics and molecular biology analysis. BSHX, a traditional Chinese medicine, suppressed multiple AGEactivated pathways, such as $\mathrm{FoxO} / \mathrm{Bim} / \mathrm{Caspase}-3$ signaling pathway to interrupt the expression of various apoptotic proteins, thereby preventing brain microvascular endothelial cells from apoptosis. The study findings suggest the potential therapeutic targets for BSHX in the management of diabetic cerebral microangiopathy and offer insights into the proteomics-guided pharmacological mechanism study of Traditional Chinese Medicine.

\section{Abbreviations}

$\mathrm{A} \beta: \quad$ Amyloid- $\beta$ protein

$\mathrm{AD}$ : Alzheimer's disease

AGEs: Advanced glycosylation end products

BP: $\quad$ The biological process

BSHX: Bushen Huoxue prescription

CC: The cellular component

DM: Diabetes mellitus

DMEM: Dulbecco's modified eagle's medium

ECM: The endothelial cell medium

FBS: $\quad$ Fetal bovine serum

KEGG: The Kyoto Encyclopedia of Genes and Genomes

LDH: The lactate dehydrogenase

MF: The molecular function

RAGE: Receptors of AGEs

ROS: Reactive oxygen species

T2DM: Type 2 diabetes mellitus.

\section{Data Availability}

The datasets used and analyzed during the current study are available from the corresponding author on reasonable request.

\section{Disclosure}

Shao-Yang Zhao, Huan-Huan Zhao should be considered co-first authors

\section{Conflicts of Interest}

The authors declare that they have no conflicts of interest.

\section{Authors' Contributions}

Shao-Yang Zhao and Huan-Huan Zhao contributed equally to this work and should be considered co-first authors. SaiMei Li and Bao-Hua Wang conceived and designed the experiments. Shao-Yang Zhao and Huan-Huan Zhao carried out the experiments and wrote the manuscript. ShaoYang Zhao and Huan-Huan Zhao analyzed the data. HuanHuan Zhao and Yi-Ming Li provided the regents/materials/ analysis tools. All the authors have read and approved the manuscript. Shao-Yang Zhao and Huan-Huan Zhao contributed equally to this work.

\section{Acknowledgments}

This work was supported by the National Natural Science Foundation of China for Youth (Nos. 82104621 and 81530099) and the Collaborative Innovation Team Construction Project of Guangzhou University of Chinese Medicine (No. 2021XK15).

\section{Supplementary Materials}

Supplementary file includes the original data of Figure 4. (Supplementary Materials)

\section{References}

[1] R. Chen, J. Shi, Q. Yin et al., "Morphological and pathological characteristics of brain in diabetic encephalopathy," Journal of Alzheimer's Disease, vol. 65, no. 1, pp. 15-28, 2018.

[2] G. T. Díaz-Gerevini, A. Daín, M. E. Pasqualini, C. B. López, A. R. Eynard, and G. Repossi, "Diabetic encephalopathy: beneficial effects of supplementation with fatty acids $\omega 3$ and nordihydroguaiaretic acid in a spontaneous diabetes rat model," Lipids in Health and Disease, vol. 18, no. 1, pp. 43-57, 2019.

[3] T. T. van Sloten, S. Sedaghat, M. R. Carnethon, L. J. Launer, and C. D. A. Stehouwer, "Cerebral microvascular complications of type 2 diabetes: stroke, cognitive dysfunction, and depression," Lancet Diabetes \& Endocrinology, vol. 8, no. 4, pp. 325-336, 2020.

[4] A. Kuhla, S. C. Ludwig, B. Kuhla, G. Münch, and B. Vollmar, "Advanced glycation end products are mitogenic signals and trigger cell cycle reentry of neurons in Alzheimer's disease brain," Neurobiology of Aging, vol. 36, no. 2, pp. 753-761, 2015.

[5] Y. Hong, C. Shen, Q. Yin, M. Sun, Y. Ma, and X. Liu, "Effects of RAGE-specific inhibitor FPS-ZM1 on amyloid- $\beta$ metabolism and AGEs-induced inflammation and oxidative stress in rat hippocampus," Neurochemical Research, vol. 41, no. 5, pp. 1192-1199, 2016.

[6] M. Guglielmotto, M. Aragno, E. Tamagno et al., "AGEs/RAGE complex upregulates BACE1 via NF-kappaB pathway activation," Neurobiology of Aging, vol. 33, no. 1, pp. 113-116, 2012.

[7] P. Nardin, C. Zanotto, F. Hansen et al., "Peripheral levels of AGEs and astrocyte alterations in the Hippocampus of STZ- 
diabetic rats," Neurochemical Research, vol. 41, no. 8, pp. 2006-2016, 2016.

[8] C. Toth, L. L. Rong, C. Yang et al., "Receptor for advanced glycation end products (RAGEs) and experimental diabetic neuropathy," Diabetes, vol. 57, no. 4, pp. 1002-1017, 2008.

[9] P. Wang, R. Huang, S. Lu et al., "RAGE and AGEs in mild cognitive impairment of diabetic patients: a cross-sectional study," PLoS One, vol. 11, no. 1, Article ID e0145521, 2016.

[10] A. M. Vincent, L. Perrone, K. A. Sullivan et al., "Receptor for advanced glycation end products activation injures primary sensory neurons via oxidative stress," Endocrinology, vol. 148, no. 2, pp. 548-558, 2007.

[11] M. A. Babizhayev, I. A. Strokov, V. V. Nosikov et al., "The role of oxidative stress in diabetic neuropathy: generation of free radical species in the glycation reaction and gene polymorphisms encoding antioxidant enzymes to genetic susceptibility to diabetic neuropathy in population of type I diabetic patients," Cell Biochemistry and Biophysics, vol. 71, no. 3, pp. 1425-1443, 2015.

[12] X. Cao, Y. Xia, M. Zeng, W. Wang, Y. He, and J. Liu, "Caffeic acid inhibits the formation of advanced glycation end products (AGEs) and mitigates the AGEs-induced oxidative stress and inflammation reaction in human umbilical vein endothelial cells (HUVECs)," Chemistry and Biodiversity, vol. 16, no. 10, 2019.

[13] L. Yuan, L. Quan, C. S. Pan et al., "Bushen Huoxue attenuates diabetes-induced cognitive impairment by improvement of cerebral microcirculation: involvement of RhoA/ROCK/ moesin and src signaling pathways," Frontiers in Physiology, vol. 9, pp. 527-540, 2018.

[14] S.-Y. Zhao, H.-H. Zhao, T.-T. Hao, W.-W. Li, and H. Guo, "Effect of bushen Huoxue prescription on cognitive dysfunction of KK-ay type 2 diabetic mice," Evidence-based Complementary and Alternative Medicine, vol. 202114 pages, Article ID 6656362, 2021.

[15] K. W. Zeng, X. M. Wang, H. Ko, H. C. Kwon, J. W. Cha, and H. O. Yang, "Hyperoside protects primary rat cortical neurons from neurotoxicity induced by amyloid beta-protein via the PI3K/Akt/Bad/Bcl(XL)-regulated mitochondrial apoptotic pathway," European Journal of Pharmacology, vol. 672, no. 1-3, pp. 45-55, 2011.

[16] B.-Y. Yan, C.-S. Pan, X.-W. Mao et al., "Icariside II improves cerebral microcirculatory disturbance and alleviates hippocampal injury in gerbils after ischemia-reperfusion," Brain Research, vol. 1573, pp. 63-73, 2014.

[17] S.-Y. Zhao, L.-X. Liao, P.-F. Tu, W.-W. Li, and K.-W. Zeng, "Icariin inhibits AGE-induced injury in PC12 cells by directly targeting apoptosis regulator Bax," Oxidative Medicine and Cellular Longevity, vol. 201912 pages, Article ID 7940808, 2019.

[18] X.-W. Zhang, S. Wang, P.-F. Tu, and K.-W. Zeng, "Sesquiterpene lactone from Artemisia argyi induces gastric carcinoma cell apoptosis via activating NADPH oxidase/reactive oxygen species/mitochondrial pathway," European Journal of Pharmacology, vol. 837, pp. 164-170, 2018.

[19] L.-C. Wang, W.-H. Wei, X.-W. Zhang, D. Liu, K.-W. Zeng, and P.-F. Tu, "An integrated proteomics and bioinformatics approach reveals the anti-inflammatory mechanism of carnosic acid," Frontiers in Pharmacology, vol. 9, pp. 370-380, 2018.

[20] K. Tsuchiya and Y. Ogawa, "Forkhead box class O family member proteins: the biology and pathophysiological roles in diabetes," Journal of Diabetes Investigation, vol. 6, no. 8, pp. 726-734, 2017.
[21] T. Zhang, D. H. Kim, X. Xiao et al., "FoxO1 plays an important role in regulating $\beta$-cell compensation for insulin resistance in male mice," Endocrinology, vol. 157, no. 3, pp. 1055-1070, 2016.

[22] K. Jash, P. Gondaliya, P. Kirave, B. Kulkarni, A. Sunkaria, and K. Kalia, "Cognitive dysfunction: a growing link between diabetes and Alzheimer's disease," Drug Development Research, vol. 81, no. 2, pp. 144-164, 2020.

[23] Y. Sun, C. Ma, H. Sun et al., "Metabolism: a novel shared link between diabetes mellitus and alzheimer's disease," Journal of Diabetes Research, vol. 202012 pages, Article ID 4981814, 2020.

[24] V. Fiore, A. De Rosa, P. Falasca et al., "Focus on the correlations between alzheimer's disease and type 2 diabetes," Endocrine, Metabolic \& Immune Disorders-Drug Targets, vol. 19, no. 5, pp. 571-579, 2019.

[25] K. Akter, E. A. Lanza, S. A. Martin, N. Myronyuk, M. Rua, and R. B. Raffa, "Diabetes mellitus and Alzheimer's disease: shared pathology and treatment?" British Journal of Clinical Pharmacology, vol. 71, no. 3, pp. 365-376, 2011.

[26] S. Davoud, G. H. Amir, A. Hassan, and A. Aslani, "Age-rage axis blockade in diabetic nephropathy: current status and future directions," European Journal of Pharmacology, vol. 833, pp. 158-164, 2018.

[27] Y. Sasai, K. Iwakawa, K. Yanagida et al., "Advanced glycation endproducts stimulate renal epithelial cells to release chemokines that recruit macrophages, leading to renal fibrosis," Bioscience Biotechnology and Biochemistry, vol. 76, no. 9, pp. 1741-1745, 2012.

[28] Z. H. Li, P. Y. Yang, and B. Feng, "Effect of atorvastatin on AGEs-induced injury of cerebral cortex via inhibiting NADPH oxidase -NF- $\kappa$ B pathway in ApoE-/- mice," Molecular Biology Reports, vol. 47, no. 12, pp. 9749-9488, 2020.

[29] Kailash and Prasad, "Age-rage stress: a changing landscape in pathology and treatment of Alzheimer's disease," Molecular and Cellular Biochemistry, vol. 459, no. 1-2, pp. 95-112, 2019.

[30] M. Zheng, Y. Xin, Y. Li et al., "Ginsenosides: a potential neuroprotective agent," BioMed Research International, vol. 201811 pages, Article ID 8174345, 2018.

[31] H. Yokota, "Applications of proteomics in pharmaceutical research and development," Biochimica et Biophysica Acta (BBA) Proteins \& Proteomics, vol. 1876, no. 1, pp. 17-21, 2019.

[32] Q.-X. Yue, Z.-W. Cao, S.-H. Guan et al., "Proteomics characterization of the cytotoxicity mechanism of ganoderic acid $\mathrm{D}$ and computer-automated estimation of the possible drug target network," Molecular \& Cellular Proteomics, vol. 7, no. 5, pp. 949-961, 2008.

[33] X. Wang, Y. Chen, Q. Han et al., "Proteomic identification of molecular targets of gambogic acid: role of stathmin in hepatocellular carcinoma," Proteomics, vol. 9, no. 2, pp. 242-253, 2010.

[34] X.-W. Zhang, X.-J. Yan, Z.-R. Zhou et al., "Arsenic trioxide controls the fate of the PML-RAR $\alpha$ oncoprotein by directly binding PML," Science, vol. 328, no. 5975, pp. 240-243, 2010.

[35] S. R. Farmer, "The forkhead transcription factor Foxo1: a possible link between obesity and insulin resistance," Molecular Cell, vol. 11, no. 1, pp. 6-8, 2003.

[36] X. Xiong, R. Tao, R. A. Depinho, and X. C. Dong, "Deletion of hepatic FoxO1/3/4 genes in mice significantly impacts on glucose metabolism through downregulation of gluconeogenesis and upregulation of glycolysis," PLoS One, vol. 8, no. 8, Article ID e74340, 2013.

[37] L. Huo, X. Bai, Y. Wang, and M. Wang, "Betulinic acid derivative $\mathrm{B} 10$ inhibits glioma cell proliferation through 
suppression of SIRT1, acetylation of FOXO3a and upregulation of Bim/PUMA," Biomedicine \& Pharmacotherapy, vol. 92, pp. 347-355, 2017.

[38] P. Sanphui and S. C. Biswas, "FoxO3a is activated and executes neuron death via Bim in response to $\beta$-amyloid," Cell Death \& Disease, vol. 4, no. 5, p. e625, 2013.

[39] T. Sun, J. Zhang, B. Deng et al., "FOXO1 and FOXO3a sensitize non-small-cell lung cancer cells to cisplatin-induced apoptosis independent of Bim," Acta Biochimica et Biophysica Sinica, vol. 52, no. 12, pp. 1348-1359, 2020. 